\title{
Bioengineering a Cadmium Sensing Green Fluorescent Protein based Whole-Cell Biosensor from Pseudomonas aeruginosa $^{\dagger}$
}

\author{
Krishani Dave 1,* \\ 1 Department of Applied Sciences, Northumbria University, City Campus, Sutherland Building, Newcastle-upon-Tyne, NE1 \\ $8 \mathrm{ST}$ \\ * Correspondence: krishani.dave@gmail.com; \\ $\dagger \quad$ Presented at International e-Conference on Bioengineering for Health and Environment (ICBHE 2020)
}

Received: 5.07.2020; Revised: 10.07.2020; Accepted: 12.07.2020; Published: 15.07.2020

\begin{abstract}
Heavy metal toxicity remediation is a major issue due to the increase in industrial waste production, with cadmium being one of the major pollutants. An approach involving homologous cloning was made to bioengineer a microbial cadmium biosensor from a strain of Pseudomonas aeruginosa. The promoter pCadR from $P$. aeruginosa was cloned into a vector pEGFP-N2. Gibson assembly cloning method was chosen considering the several advantages it holds over the traditional cloning techniques. Escherichia coli DH5 alpha strain was used as the host cell, which on sensing cadmium, produced fluorescence using the reporter gene called green fluorescent protein (GFP). The clone, pEGFP-N2 CadR, was subjected to increasing concentrations of cadmium chloride to determine the sensitivity. It was observed that pEGFP-N2 $2_{\text {CadR }}$ responded well to micromolar concentrations of cadmium chloride, and hence it may be used to detect low concentrations of cadmium. To determine the specificity of the sensor, it was also tested with lead nitrate and copper sulfate solutions. It was determined that the biosensor had non-specific interactions with other metals. The interaction of the promoter with the metals was weak as compared to previous studies, and this was attributed to slight variations and mutations observed in the promoter sequence following subsequent rounds of sequencing. As the fluorescent intensities of the sensor in response to the metals were not highly distinguishable, this biosensor was not classified as an effective cadmium sensor. Further studies for determining the promoter interaction and affinity towards various metals at varying concentrations are required to validate the results obtained.
\end{abstract}

Keywords: Heavy metal toxicity; biosensor; clone; Pseudomonas aeruginosa.

(C) 2020 by the authors. This article is an open-access article distributed under the terms and conditions of the Creative Commons Attribution (CC BY) license (https://creativecommons.org/licenses/by/4.0/).

\section{Funding}

This research received no external funding.

\section{Acknowledgments}

This research has no acknowledgment.

\section{Conflicts of Interest}

The authors declare no conflict of interest. 